Thirteenth International Conference on Magnet Technology, Victoria B.C. " BNL-49551 Canada, September 20-24, 1993

\title{
MAGNETIC PERFORMANCE OF A VARIABLE PERIOD, FAST EXCITATION, WIGGLER*
}

\author{
J. Gallardo, T. Romano, A. van Sieenbergen \\ Pliysics Depl. and NSLS Dept., Brookhaven National Laboratory. Upton NY 11973
}

\begin{abstract}
With the objective of carrying out an Inverse Free Electron Laser (IFEL) electron accelerator experiment, an iron dominated (Vanadium Permandur) fast excitation, laminated (variable periad length) planar wiggler, making use of copper field reflectors, has been constructed and has been measured in several period length tapering configurations. This report presents an analysis and experimental results of this wiggler, typically of which the parameters are $\lambda(w)=3.7$ $\mathrm{cm}, \mathrm{B}(\max )=1.8 \mathrm{~T}$.
\end{abstract}

\section{IntRODUCTION}

The study of the INVERSE FREE ELECTRON LASER (IFEL), as a potential mode of electron acceleration has been pursued at Brookhaven National Laboratory for a $\cdots:$ : number of years. More recent studies focussed on the development of a low energy (few GeV), high gradient, multistage lineat accelerator[1]. Specifically also, the design of a short accelerator module has been pursued which will make use of the $50 \mathrm{MeV}$ Linac beam and high power ( $210^{11}$ Watts) $\mathrm{CO}_{2}$ laser beam of the Accelerator Test Facility (ATF), Center for Accelerator Physics (CAP) of Brookhaven National Laboratory; and a fast excitation pulsed electromagnetic wiggler. In the IFEL accelerator mode the electron beam is accelerated through its interaction with the high power laser radiation in the medium of the periodic wiggler field. For acceleration it is necessary to maintain synchronism for the propagating electron beam and radiation wave, hence for a laser field with constant $\lambda$ (laser) it is necessary to "taper" the wiggler. This taper can be accomplished while holding the wiggler parameter $K_{w}\left(=93.4 B \lambda_{w}[T m]\right)$, the period length $\lambda_{w}$. of the maximum wiggler field $B_{w}$ constant. The choice is restricted by the maximum practical wiggler field and the minimum wiggler period length. It has been shown[2] that the maximum rate of acceleration, averaged over the full accelerator length, is obtained for a constant $B_{w}$ accelerator. Hence, for the IFEL accelerator the use of a period length tapered wiggler, maintaining $B_{w}$ constant has been adopted. Although such a wiggler structure could be constructed using permanent magnets, the requirement of a specific period length taper would be costly and also difficult to subsequently change, if need be. Instead. the destin and construction of a laninated, fast excitation eleviromagnetic wiggler has been pursued[3], [4] which permuls readyrvariation of the wiggler period length taper. Thıs wggler consists of stackable, geometrically altematng substacks of identical ferromasnetic (Vanadium

\footnotetext{
Work pirivmisd under the auspices of the U.S. Deparment of Eneroy"

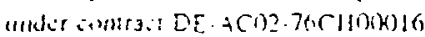

Permandur [VaP]) laminations, assembled, in $\left(\lambda_{w} / 4\right)$ thickness substacks, separated by nonmagnetic laminations (Fig. 1,2). Four straight conductive rods, parallel to the axis and interconnected only at the ends of the assembly, constitute the single current loop that drives the wiggler. The stacks are easily assembled, are compressed by simple tie rods, and allow ready variation of wiggler period taper. A dramatic improvement in maximum achievable wiggler field, $B_{w}$, results from uring conductive material for the nonmagnetic laminations, so that the induced fields from the eddy currents uncouple the wiggler "up" field from the "down", field. : These, effectively "field reflectors", enhance significantly the maximum achievable field on axis as shown in Fig. 3, where $B_{w}$ vs I(excit.) is given (wiggler gap $=4 \mathrm{~mm}$ ). Field saturation is evident in these results for higher excitation current values. The onset of field saturation is clearly discernable with the onset of distortion of the magnetic measurement probe voltage versus time display. . The field value corresponding to the onset of saturation for a sequence of model measurements with different period length values, was obtained both for the case of wiggler models without field reflection and with field reflection.

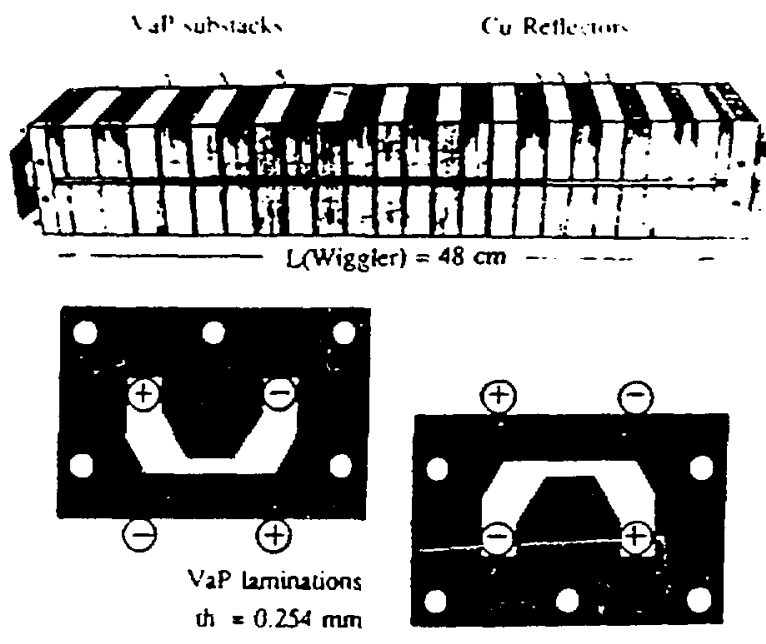

Fig.l

Fast Excilation, Variable Pcriod, Wiggler [IFEL Accelcrator, Wiggler Piotolype] Stakikable, Interlcaved VaP laminations and Cu eddy' current "fickd refleciors" 
This is shown in Fig. 4 clearly indicating the effectiveness of the field reflectors for this type of fast excitation (pulse length $200 \mu \mathrm{sec}$ ) pulsed wiggler magnet.

Further characterization of this wiggler is provided in Section II where the basic analytical field expressions are derived, and in Section III where detailed field plots and related parameters for a constant period wiggler and a tapered period wiggler are presented.

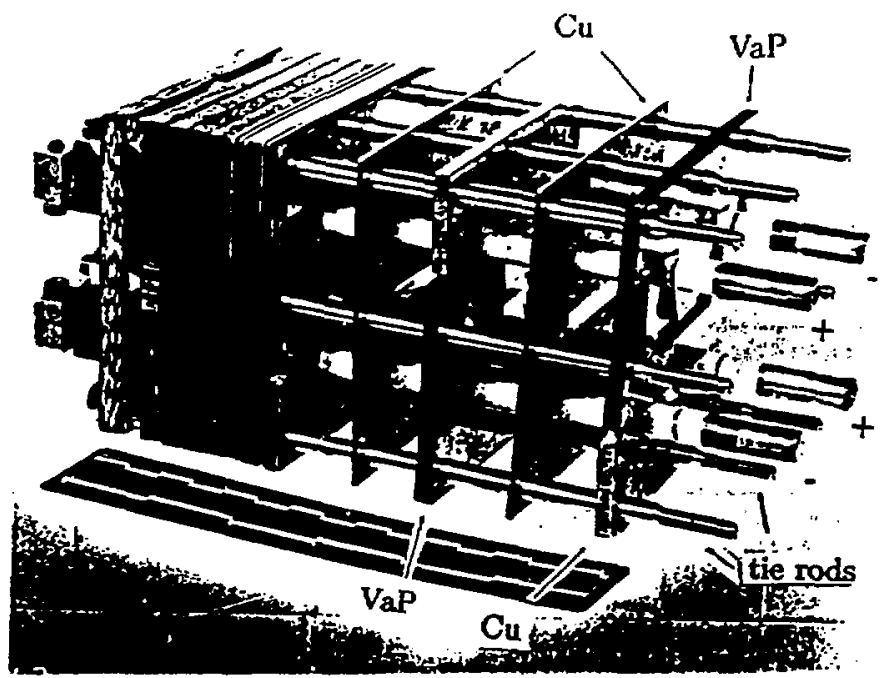

Fig.2

"Exploded View" Fast Excitation Variable Period Wiggler [Stackable Laminated Structurel

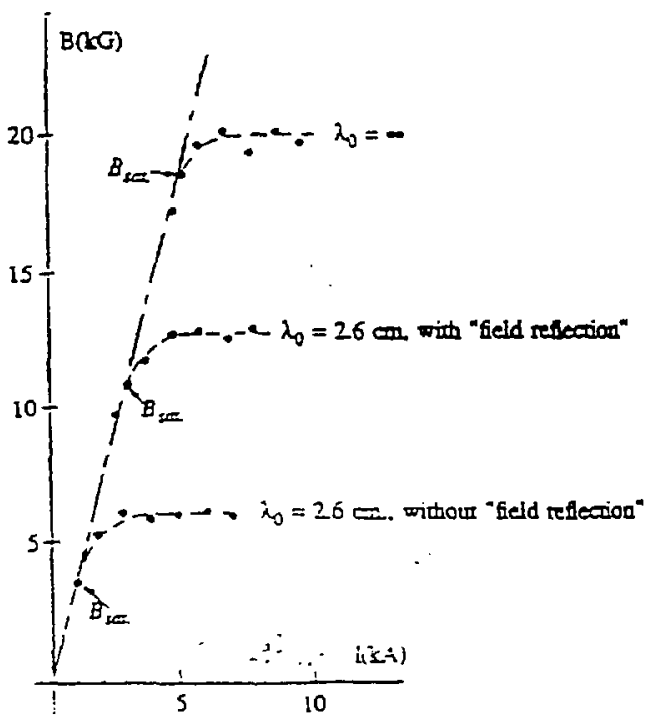

Fig.3

Fast Exciation Vivgher, B is I

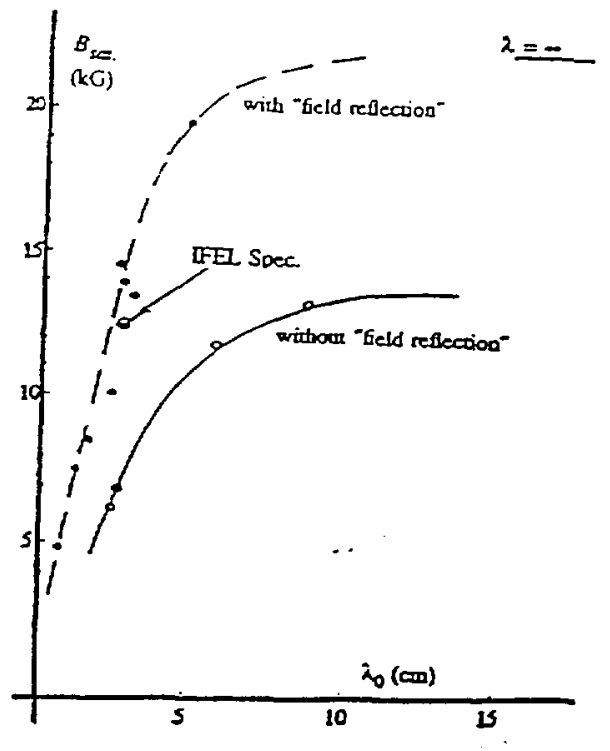

Fig.4

Fast Excitation Wiggler, $B_{\text {sce. }}$ vs $\lambda_{0}$ $\mathrm{Gap}=4.0 \mathrm{~mm}$

\section{WIGGLER FIELD}

For the IFEL interaction to take place it is required that electron beam and radiation wave effectively overlap, hence the precise trajectory of the electron beam in the body of the wiggler need to be deduced from actual wiggler fields. Hence, it is necessary to obtain a complete reconstruction of the field in the wiggler gap from the measured field values. From general principles[5] the magnetic scalar potential is expressed as:

$$
\phi(x, y, z)=X(x) Y(y) Z(z)
$$

with $\bar{B}(x, y, z)=-\bar{\nabla} \Phi(x, y, z)$.

If symmetry is assumed in the horizontal plane and periodicity in the longitudinal direction then $X(x)=X(-x)$ and $Z(z)=\cos$ akz where $k=2 \pi / \lambda$ with $\lambda$ the period, the most general expression for the scalar potential can be written as:

$$
\phi(x, y, z)=\sum_{a=1}^{-} \cosh \left(n k_{2} x\right)\left[A_{\alpha} \exp \left(n k_{2} y\right)+B_{a} \operatorname{cxp}\left(-n k_{2} y\right)\right] \cos \left(n k_{3} z\right)
$$

with the constraint $k_{3}{ }^{2}=k_{1}{ }^{2}+k_{2}{ }^{2}=k_{2}{ }^{2}\left(1+r^{2}\right)$.

Notice that there is no median plane symmetry and the deviation can be represented with a single parameter $\eta$ such that,

$$
B_{y}(x,-g, z)=\eta B_{y}(x, g, z)
$$

where $g$ is the gap magnitudi. This relation can be understood making use of the Gauss theorem in the transverse plane (see Fig. I). 
Simple algebraic manipulations lead to the expression of the field in the gap, as follows:

$$
\begin{aligned}
& B_{n}(x, y, z)=\sum_{n=1}^{-} n k_{1} \sinh \left(n k_{1} x\right)\left[A_{n} \exp \left(n k_{2} y\right)+B_{n} \exp \left(-n k_{2} y\right)\right] \cos \left(n k_{3} z\right) \\
& B_{y}\left(x_{2} y, z\right)=\sum_{n=1}^{\infty} n t_{2} \cosh \left(n k_{1} x\right)\left[A_{n} \operatorname{cxp}\left(n k_{2} y\right)+B_{n} \operatorname{cxp}\left(-n k_{2} y\right)\right] \cos \left(n k_{3} z\right) \\
& B_{z}\left(x_{n} y, z\right)=-\sum_{n=1}^{-} n k_{3} \cosh \left(n k_{1} x\right)\left[A_{n} \exp \left(n k_{2} y\right)+B_{n} \exp \left(-n k_{2} y\right)\right] \sin \left(n k_{2} z\right)
\end{aligned}
$$

The coefficients $A_{n}$ and $B_{n}$ are determined by the boundary conditions at the poles and the peak vertical magnetic field on axis $\mathrm{B}_{\text {on }}$; it follows:

$$
\begin{aligned}
& A_{n}=\frac{B_{e n}}{2 n k_{2}(1+\eta) \sinh \left(n k_{2} d\right)\left[\operatorname{cxp}\left(n k_{2} d\right)-\eta \exp \left(-n k_{2} d\right)\right]} \\
& B_{a}=\frac{B_{o n}}{2 n k_{2}(1+\eta) \sinh \left(n k_{2} d\right)\left[\exp \left(-n k_{2} d\right)-\eta \exp \left(n k_{2} d\right)\right]}
\end{aligned}
$$

With the fields so determined the electron trajectory in the wiggler field can be calculated.

\section{Performance Of THE FAST EXCTTATION WIGGLER}

Measured field values $B_{0}(z)$ vs $z$, on axis, for an excitation current of $6 \mathrm{kA}$, are given in Fig. 5 . The rmsfluctuation of the peak field $\sigma_{\mathrm{B}}=\sqrt{ }<\delta \mathrm{B}^{2} / \mathrm{B}^{2}>$ about the average is under $0.2 \%$ and the maximum scatter is 1.0\%. A complete measured field representation $B_{y}(x, y, z)$ for a single pole region ( $g=4 \mathrm{~mm}, 0.5 \lambda=18.8 \mathrm{~mm}, I$ $=6 \mathrm{kA}$ ) is given in Fig. 6 for $-1.2 \leq y \leq 1.2 \mathrm{mmm}$ and $-6.0 \leq x \leq 6.0 \mathrm{~mm}$. A Fourier transform analysis of the entire field map on axis has been performed. The power spectrum is shown in Fig. 7. From the power spectrum plot, the odd harmonics up to order 7th are clearly in evidence, a natural consequence of the periodicity of the field. The magnitude of the noise level is about $10^{-6}$ and up to that level there is no indication of even harmonics. The 3rd. harmonic is about $3 \%$ of the fundamental. The normalized horizontal velocity $x^{\circ}=v_{x} / v_{z}$ is given essentially by the first integral of the vertical component of the magnetic field $B_{y}(0,0, z)=B_{o}(z)$, i.e.,

$$
x^{\prime}=\frac{K 2 \pi}{\gamma} \int_{-\infty}^{z} d z^{\prime} \frac{B_{0}(z)}{B^{\max }}
$$

Similarly so, the position $x$ is given by the second integral of lie field as,

where $K=\mathrm{eB}^{\max } \lambda_{u} / \mathrm{nx}^{2}-\beta, 2 \pi=0.934 \mathrm{~B}^{\mathrm{mix}}|\mathrm{T}| \lambda_{u} \cdot|\mathrm{cm}|$,

$$
x=\frac{K}{\gamma} \frac{2 \pi}{\lambda_{w}} \int_{-\infty}^{z} d z^{\prime}\left(z-z^{\prime}\right) \frac{B_{0}\left(z^{\prime}\right)}{B^{\max }},
$$

with $B^{\max }-1.4 \mathrm{~T}$ and $\lambda_{w} \simeq 3.754 \mathrm{~cm}$. The magnitudes of $x$ vs $z$ and $x^{\prime}$ vs $z$ are shown in Figs. 8 and 9, respectively. As evident, the maximum $x$ oscillation amplitude is $\approx 0.4 \mathrm{~mm}$. The displacement and steering of the beam at the exit of the wiggler are approximately $0.001 \mathrm{~mm}$ and $3.4 \mathrm{mrad}$, respectively.

For completeness sake a plot of the field $B_{0}(z)$ vs $z$ for a tapered period wiggler with $2.98 \mathrm{~cm}<\lambda_{w}<5.04 \mathrm{~cm}$ is shown in Fig. 10.

\section{CONCLUSION}

It has been shown that the fast excitation driven, laminated Vanadium Permendur wiggler with periodic interleaving of conductive copper field reflectors is capable of satisfying both the FEL and IFEL requirements. As indicated, a specific tapered period slope can readily be achieved. The wiggler structure, as presently executed is robust and simple to assemble and lends itself for easy modifications of period length, field magnitude and taper. These are very important features in actual FEL and IFEL experiments.

\section{ACKNOWLEDGEMENTS}

The authors wish to acknowledge the contribution of $M$. Woodle and $\mathrm{J}$. Armendariz to the experimental work. They are also grateful to $L$. Gianessi for making the code SIN-LUCE available. This work was supported by the Advanced Technology R\&D Branch, Division of High Energy Physics, U.S. Dept. of Energy.

\section{REFERENCES}

[1] A. Fisher, 1. Gallardo, J. Sandweiss, A. van Steenbergen, -Inverse free electron la ser accelerator, III Workshop on Adv. Accel. Concepts, Pon Jefferson, NY, June 1992.

[2] A. van Sieenbergen, "Experimental program, ATF", BNL 41664, (1988).

[3] A. van Sicenbergen, J. Gallardo, T. Romano, M. Woodle, "Fast excication wiggler development". Workshop on a 1 Angstrom FEL, Sag Harbor, NY, BNL 52273, 1990.

[4] A. van Steenbergen, US Patent Apptication 368618, June 1989, issued Aug. 1990.

[5] G. Dstoli, A. Renieri, Exp. and Theor. Aspects of the FEL, "Laser Handbook, Vol. 4, North Holland, Amsterdam, (1985). 


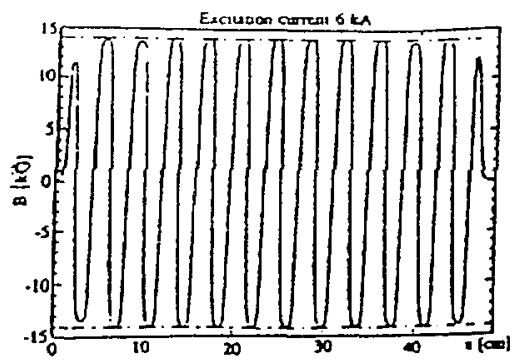

Fig.s

Constant Period Wiggler, $B_{y}$ vs $z$ $\lambda=3.76 \mathrm{~cm}, \mathrm{~g}=4.0 \mathrm{~mm}$

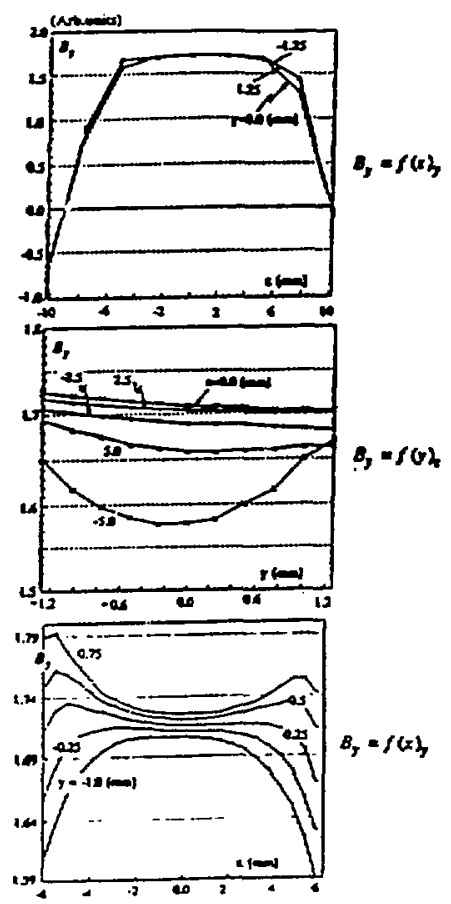

Fig.6

Wiggler Field Distribution

$\lambda=3.76 \mathrm{~cm}, \mathrm{~g}=4 \mathrm{~mm}, I_{\mathrm{ex}}=6 \mathrm{kA}$

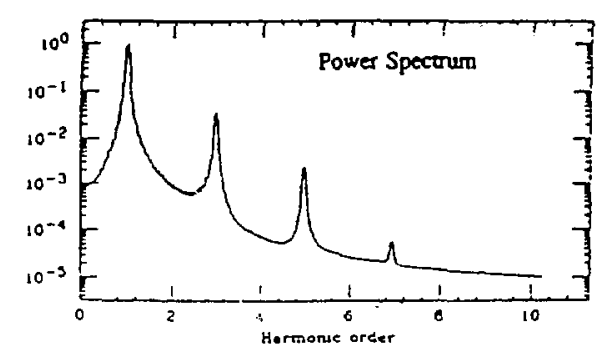

$\therefore-\therefore$ Fig.7

Power Spectrum, Constant Period Wiggler $\lambda=3.76 \mathrm{~cm} . g=4.0 \mathrm{~mm}, I(\mathrm{exc})=6 \mathrm{kA}$

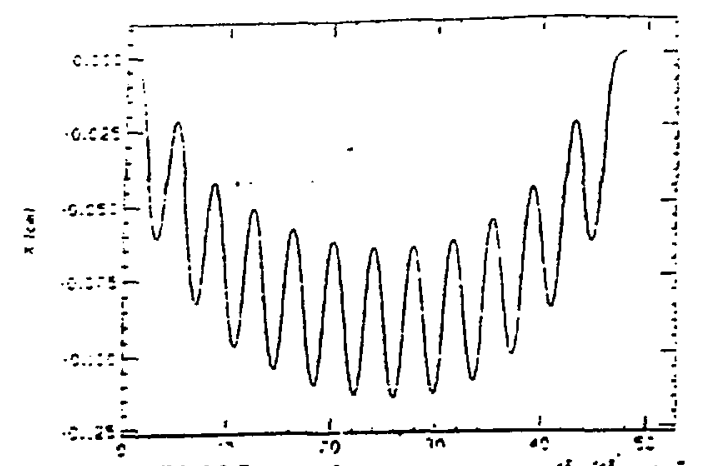

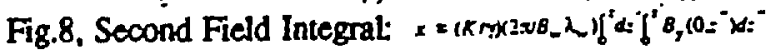
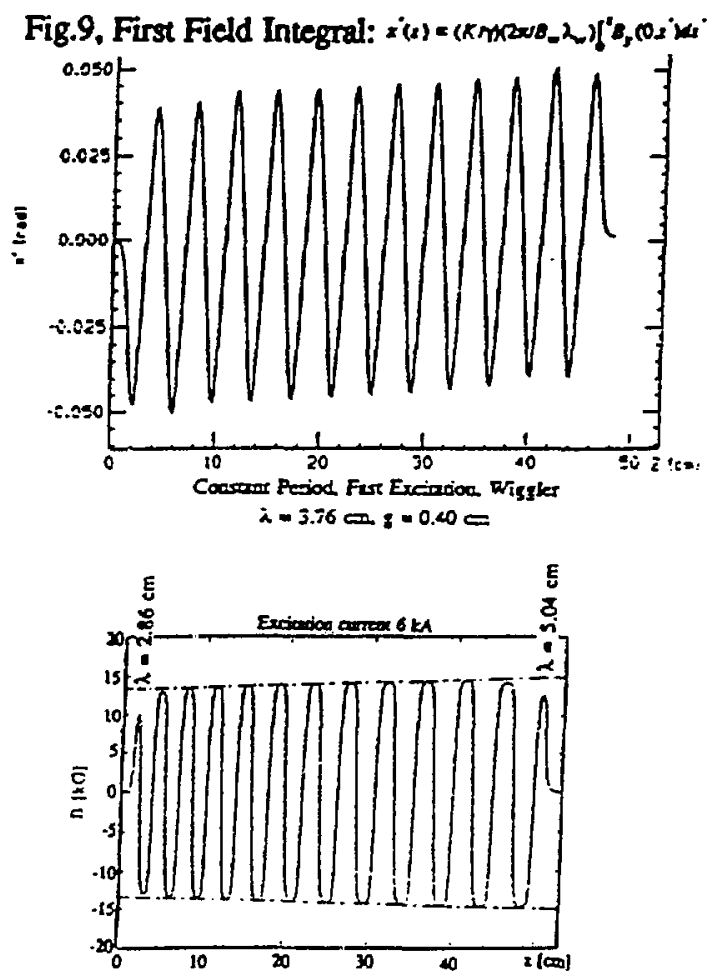

Fig. 10

Variable Period, Fast Excitation, Wiggler (IFEL Accelerator, Wiggler Prototype) $\lambda=2.98-5.04 \mathrm{~cm}, d \lambda d z=4 \%, g=4.0 \mathrm{~mm}$

\section{DISCLAIMER}

This report was prepared as an account of work sponsored by an agency of the United States Government. Neither the United States Government nor any agency thereof, nor any of their employees, makes any warranty, express or implied, or assumes any legal liability or responsibility for the accuracy, completeness, or usefulness of any information, apparatus, product, or process disclosed, or represents that its use would not infringe privately owned rights. Reference herein to any specific commercial product, process, or service by trade name, trademark, manufacturer, or otherwise does not necessarily constitute or imply its endorsement, recommendation, or favoring by the United States Government or any agency thereor. The views and opinions of authors expressed herein do not necessarily state or reflect those of the United States Government or any agency thereof. 